\title{
Design and Implementation of Automatic Emergency Braking System
}

\author{
Sandeep Thorat*, SanketThorve, Jaydatta Upase and Agampal Singh Dhupar \\ ${ }^{1}$ Mechanical Department, M.I.T College of Engineering, University of Pune, Pune, India
}

Accepted 02 March 2016, Available online 15 March 2016, Special Issue-4 (March 2016)

\begin{abstract}
Vehicle technology has increased rapidly in recent years, particularly in relation to braking systems and sensing systems. ASS (active safety systems) are being researched and developed to prevent accidents and target mitigation. Among many useful active safety systems, it has been reported that AEBS (Advanced Emergency Braking Systems) effectively prevents accidents and reduces casualties simultaneously. The project aims to distinguish between systems currently in production like traction control (TC), electronic brake force distribution (EBD), brake assist (BA) and electronic stability control (ESC) functions and future systems that are currently in development. The project aims to develop a prototype system that offers a collision functionality in production vehicle, a system which can operate automatically with the help of high profile sensors based relay circuit and some modification in traditional braking system that can alert the driver in front collision and apply the brake automatically in emergency or critical situation.
\end{abstract}

Keywords: ASS (active safety systems),AEBS (Advanced Emergency Braking Systems), brake assist (BA), electronic stability control (ESC)

\section{Introduction}

Driving is a compulsory activity for most people. People use their car to move from one place to other place. The number of vehicles is increasing day by day. Nowadays, accidents are increasing and are uncertain. Accident will occur every time and everywhere and cause worst damage, serious injury and even death. These accidents are mostly caused by delay of the driver to hit the brake. This project is designed to develop a new system that can solve this problem where drivers may not brake manually but the vehicles can stop automatically by detecting obstacles. This project is about a system that can control braking system for safety. Using ultrasonic as a ranging sensor, its function based on ultrasonic wave. After transmitting by transmitter, the wave can reflect when obstacle is detected and then received by receiver. The braking circuit's function is to slow down or stop the car automatically after receiving signal from the sensor.

The objectives of this project are

i. To develop a safety car braking system using ultrasonic photoelectric retro reflective sensors.

ii. To design a vehicle with less human attention to the driving

*Corresponding author: Sandeep Thorat
Scope of project is to develop an ultrasonic sensor to detect the obstacle and to process the output from the ultrasonic sensor and to drive the pneumatic cylinder as an actuator.

\section{Literature Review}

\subsection{Safety Consideration in Cars}

There are various safety consideration involve to make passenger comfortable while traveling, and it has been modified as generation of automobile goes by. Here are some area the modification has been take place:

\subsubsection{Strong Chassis}

In old days the chassis were made of wooden floor and not good enough to sustain the load. But now days the chassis is made of alloy of lightweight aluminum and strong cast iron to give chassis immense strength such that it withstand load and sustain in accident.

\subsubsection{Dash Board Modification}

The Dash board is modified and simplified in recent days such that it can be user friendly. Ergonomics (the relation between man \& machine) of dash board include various sensors and meters to make it comfortable. 


\subsubsection{Implementation of Air Bags}

Airbags (passive restraints) have saved many lives and reduced injuries in vehicle crashes, there are accidents where they have been the cause of injury and/or death. Knowledge of the history of airbag systems and how they work is important in understanding if they are a factor in an occupant's injury or death. An overview of these subjects is presented in this paper. In addition, potential airbag defects are defined.

\subsubsection{Power Braking, ABS, Power starring, TRC}

Today many path braking technologies and brake components such as brake boosters, brake cylinders, brake hydraulic valve, brake lines, etc., lend a braking system high efficacy. They are also the key behind advanced braking systems such as the anti-lock braking system. The following are some braking systems and their components.

\subsection{Types of Brakes}

\subsubsection{Disc Brakes}

Disc brakes make use of friction generated between the disc attached to a heel and the wheel itself, to slow or stop an automobile. Disc brakes comprise brake pads that serve as friction material and are fixed onto a device called a brake caliper. When the brake pedal is pushed by a driver the brake pads are mechanically, hydraulically, pneumatically or electromagnetically forced against both sides of the disc mounted on wheels thus regulating the speed of the vehicle or slowing down the vehicle.

\subsubsection{DrumBrakes}

Louis Renault is credited for inventing the modern drum brakes in 1902 to regulate speed by causing friction between the wheels and a set of shoes or pads that push against the inside surface of a rotating drum. The drum is attached to the rotating wheels. Typically, drum brakes are classified as either leading/trailing or twin leading. Out of the two types, the twin leading drum brakes are more effective.

\subsubsection{Hand Brakes}

Hand brakes are latching brakes and are normally used to keep an automobile stationary, when not being driven. Also known as e-brake, emergency brake, park brake, parking brake or slide stick, the brakes are often configured on the floor and between front passenger and the driver. However, they can even be configured as a lever at the bottom of the dashboard, or as a footoperated pedal. The brake comprises a cable that connects the braking system at one end and a lever on the other. The driver operates the hand-lever (or a pedal) to actuate or release the brake.

\subsubsection{Power Brakes}

A braking system employing power braking uses the engine's power and/or the power of batteries to assist the driver in braking. Although conventional brakes generate enough force to regulate the speed of an automobile, power brakes further enhance this power by supplementing it from other sources (i.e. engine/batteries), thus causing highly efficient braking. Examples: air suspended brakes, vacuum suspended brakes, hydraulic booster and electro-hydraulic boost brakes.

\subsubsection{HydraulicBrakes}

Hydraulic brakes consists of a braking mechanism that uses brake fluid, to apply the braking force from the controlling unit (viz. brake pedal) to the actual brake mechanism. They are based on a multiple piston system, wherein the brake pedal when pushed, produces an equivalent force on each of the output pistons, thus multiplying the force and cause efficient braking.

\subsection{Rear End Collisions}

Three databases from three different countries are used as the basis for the problem definition to find types of collisions. Volvo's statistical accident database contains Volvo vehicles in Sweden in which the repair cost due to an accident exceeds a specified level, currently SEK 45000 . The database, which contains information about the crash, the vehicles and the occupants including injuries if any, is further described. The GIDAS database (German In-Depth Accident Study) is the second European database used in this study.

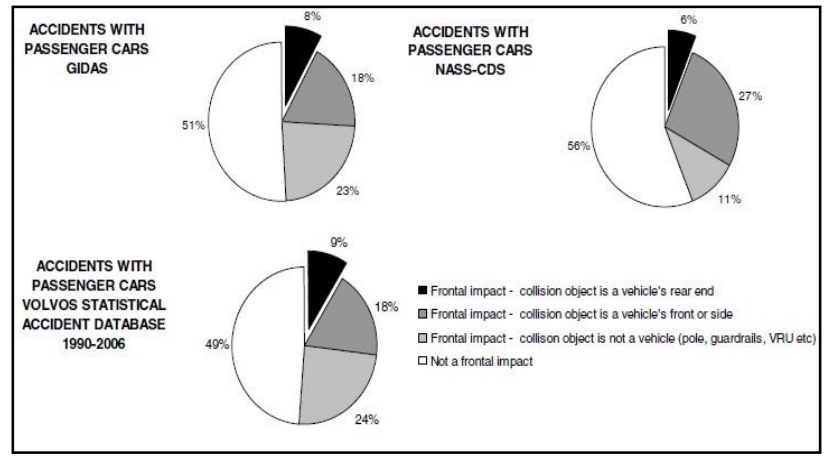

Fig.1 : Distribution of impact for GIDAS, NASS-CDS and Volvo Statistical Accident Databases

Traffic accidents within Hanover and Dresden and the rural areas surrounding these cities are investigated according to a statistical sampling process. As a complement to the European data, NASS/CDS (National Automotive Sampling System Crashworthiness Data System) is also used. CDS provides in-depth crash investigations of a representative sample of police-reported tow-away 
crashes throughout the United States. Data is weighted to provide a nationwide estimate of all types of crashes and injuries. Focusing on light vehicle crashes in the NASS/GES database, show that rear-end collisions are most frequent among all crash types accounting for $29 \%$ of all crashes. Approximately $50 \%$ of all impacts are to the front of the vehicle. Frontal impacts into an opponent motor vehicle's rear end account for 6-9\% of the total share.

In Germany approximately $12 \%$ of all accidents with persons injured and approximately $20 \%$ of all material damage accidents are caused by cars in rear end collisions. As a consequence, Bosch is introducing collision avoidance and mitigation systems for rear impact scenarios. Warning, brake support, and autonomous emergency braking are part of Bosch's Advanced Emergency Braking Systems which address such accidents. This study determines the benefit of these assistance and safety systems and estimates the collision avoidance capability considering the driver's behavior. By analyzing representative accidents with injuries from the GIDAS (German In-Depth Accident Study) database, a high potential for collision warning and avoidance systems was determined. For the first time in such a study, this analysis considers the effects of different driver reactions due to warning, braking support, or autonomous braking with respect to the possible driver behavior.

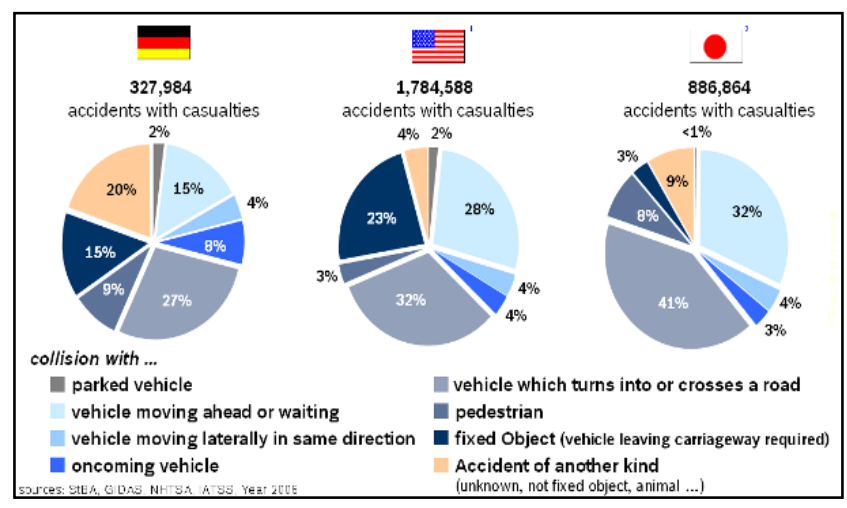

Fig.2: Accidents with casualties by kind of accident

As proposed in the NHTSA review for the New Car Assessment Program (NCAP) from July 2008, new test requirements will be introduced for Forward Collision Warning (FCW) systems. Currently there are three test scenarios defined although two scenarios are in focus of the discussion:

- 1st scenario: Subject vehicle approaches a stopped principle other vehicle at $45 \mathrm{mph}(72.5 \mathrm{kph})$. The system must give a warning $2.7 \mathrm{~s}$ prior to collision.

- 2nd scenario: Subject vehicle follows principle other vehicle at $45 \mathrm{mph}(72.5 \mathrm{kph})$. The other vehicle starts braking. The system must give a warning $2.4 \mathrm{~s}$ prior to collision.

- 3rd scenario: Subject vehicle at $45 \mathrm{mph}$ (72.5kph) encounters a slower principle other vehicle with speed
$20 \mathrm{mph}(32.2 \mathrm{kph})$. The system must give a warning at 2.1s prior to impact.

\section{Working Principle}

The system is consist of two photoelectric distance measurement sensors, kit of electronic control unit to give input and output of sensor, hydraulic circuit and single acting hydraulic cylinder. The sensor is having 15 volt supply and can measure hurdle upto $10 \mathrm{~m}$.The Brake Kit is going to be fabricate having redesign brake paddle with reduced mass. The hydraulic cylinder we are using is single acting spring return type having solenoid valve.

\section{Procedure}

Let us Consider that A Vehicle Speeding at $25 \mathrm{Km} / \mathrm{h}$ Constantly and think worst case that Driver is Fallen asleep While Driving And Vehicle is Approaching to a park car at same speed, so here is how the Auto brake System will Work

1. The Photo electric Sensor Which will sense the any object in Front and Will give Continues output while on go ,in this case if car in front comes in contact with the sensor it will give output to the Electronic Control Unit at $10 \mathrm{M}$ Distance remaining.

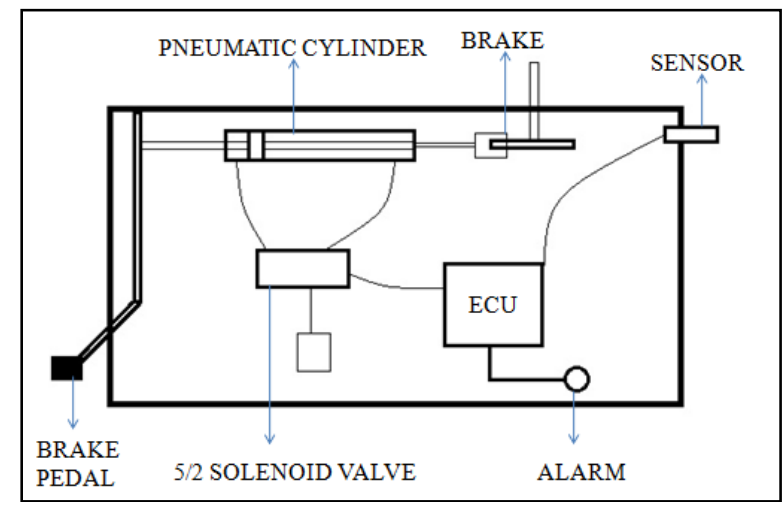

Fig.3: Concept Diagram

2. Thus ECU automatically alert the driver by blowing the alarm, thus if Driver here the alarm, the Distraction phase of Driver such that (Sleeping while Driving, Use of Mobile, Gossiping or doing any other Activity)will come to end and he will take control of the situation and further damage can be Prevented.

3. If Driver does not Respond to this Alarm mean that no action is implies to stop the vehicle then ECU will send the signal to hydraulic circuit.

4. Thus Stroke Of Hydraulic cylinder will start and lead to Auto Forcing the Brake Paddle to engage Position and lead to stopping the Vehicle, if in case Driver reaction lead to take control the situation, Driver will apply the force on Paddle then the Connection Between the Piston And Brake Paddle Will Disengage and Paddle will Force Down. 
5. Thus, after stopping the vehicle, spring will force the paddle to take its original position and thus returning it will connect to the piston help to reposition the stroke. 6. Thus brakes will apply the normal way as well as in distraction situation accident can be prevented by reducing the reaction time.

\subsection{Working Prototype}

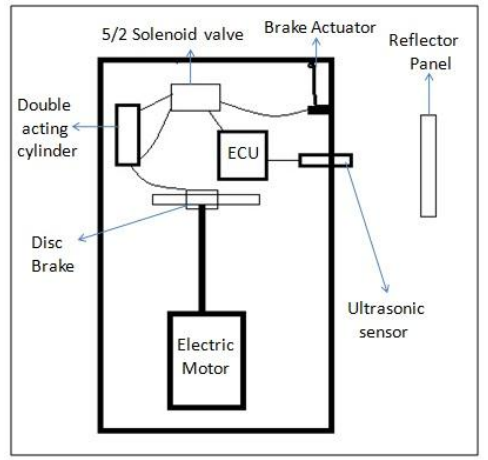

Fig:4: Prototype

Components Used:

1. Braking kit of motorcycle: It consists of disc brake driven by motor and brake calipers of a motorcycle to reduce the cost of prototype.

2. 5/2 solenoid valve: Solenoid valve is used to bypass the braking circuit to actuate the brakes

3. Ultrasonic sensor: It is used to detect objects or obstacles upto a range of $10 \mathrm{~m}$

4. Reflector

5. PLC Circuit: Ladder logic relays to actuate the alarm and actuate the solenoid valve

6. Alarm or hooter

\subsection{Solenoid Valve}

The control valve is used to control the flow direction is called cut off valve or solenoid valve. This solenoid cut off valve is controlled by the emergency push button. The solenoid valve consists of electromagnetic coil, plunger and spring. The air enters to the pneumatic solenoid valve when the push button is in ON position. A plunger connects the closure device to the control element of the actuator. The spring acting on the control element forces the closure device down into the closed position on the valve seat. The pilot supply overcomes the spring force to lift the control element into the open position. These valves are mainly suitable for contaminated or extremely viscous process fluid. In this project we are supplying the signal to solenoid valve through ECU.

\subsection{Pneumatic Cylinder}

Both single acting as well as double acting cylinder can be used, accordingly solenoid valve vary. Generally $3 / 2$ solenoid valve for the single acting cylinder and $5 / 2$ solenoid valve for the double acting cylinder are use.
The compressed air from the compressor is passed through the regulator which is able to provide the required amount of pressure by adjusting it. There is pressure gauge arrangement on the compressor which is helpful for providing the required amount of pressure easily. This compressed and pressure regulated air is then passed through the solenoid valve. If solenoid valve is $3 / 2$ then there will be only one outlet and if it is $5 / 2$ then there will be two outlets for single inlet. This outlet from solenoid valve is/is directly connected to the pneumatic cylinder with the help of hose.

\subsection{The Fundamental of Sensor}

Sensor is an electrical device that maps an environmental attribute to a quantitative measurement. It is created to collect information about the world. Each sensor is based on a transduction principle which is conversion of energy from one form to another form.

\subsubsection{The Fundamental of Ultrasonic Sensor}

Ultrasonic ranging and detecting devices use highfrequency sound waves to detect the presence of an object and its range. The systems either measure the echo reflection of the sound from objects or detect the interruption of the sound beam as the objects pass between the transmitter and receiver.

An ultrasonic sensor typically utilizes a transducer that produces an electrical output in response to received ultrasonic energy. The normal frequency range for human hearing is roughly 20 to 20,000 hertz. Transducers are devices that convert electrical energy to mechanical energy, or vice versa. The transducer converts received echoes into analog electrical signals that are output from the transducer.

The piezoelectric effect refers to the voltage produced between surfaces of a solid dielectric (nonconducting substance) when a mechanical stress is applied to it. Conversely when a voltage is applied across certain surfaces of a solid that exhibits the piezoelectric effect, the solid undergoes a mechanical distortion.

Such solids typically resonate within narrow frequency ranges. Piezoelectric materials are used in transducers, e.g., phonograph cartridges, microphones, and strain gauges that produce an electrical output from a mechanical input. They are also used in earphones and ultrasonic transmitters that produce a mechanical output from an electrical input. Ultrasonic transducers operate to radiate ultrasonic waves through a medium such as air. Transducers generally create ultrasonic vibrations through the use of piezoelectric materials such as certain forms of crystal or ceramic polymers.

\subsubsection{Measurement Principle/Effective Use of Ultrasonic Sensor}

Ultrasonic sensor transmits ultrasonic waves from its sensor head and again receives the ultrasonic waves 
reflected from an object. By measuring the length of time from the transmission to reception of the sonic wave, it detects the position of the object.

\subsubsection{The advantages of Ultrasonic Sensor}

i. Discrete distances to moving objects can be detected and measured.

ii. Less affected by target materials and surfaces, and not affected by color.

Solid-state units have virtually unlimited, maintenance free life. Ultrasonic can detect small objects over long operating distances.

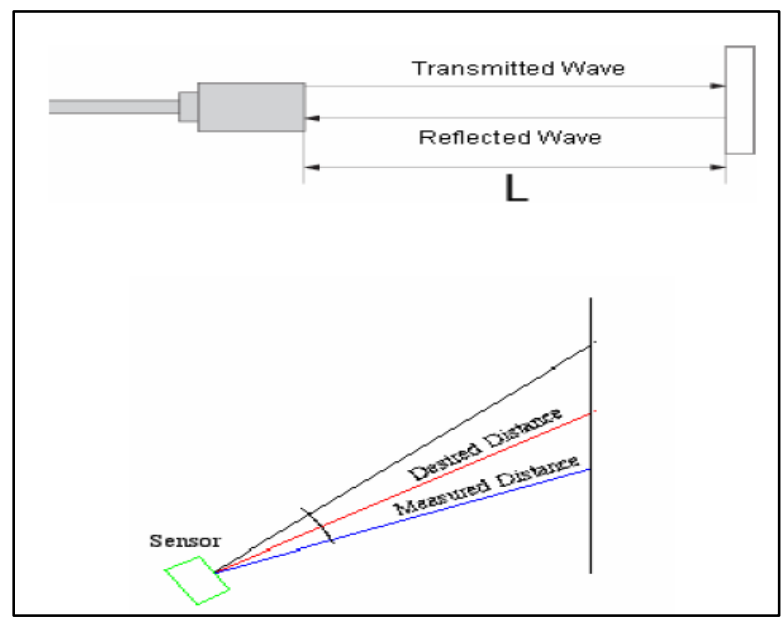

Fig.5: Schematic of working of ultrasonic sensor

\subsubsection{The Disadvantages of Ultrasonic Sensor}

i. Overheating of a wave emitter precludes the energy of ultrasonic waves emitted there from being enhanced to a practical level.

ii. Interference between the projected waves and the reflected waves takes place, and development of standing waves provides adverse effects.

iii. It is impossible to discern between reflected waves from the road surface and reflected waves from other places or objects.

\section{Case Study on AEBS}

\subsection{Test Study on AEBS}

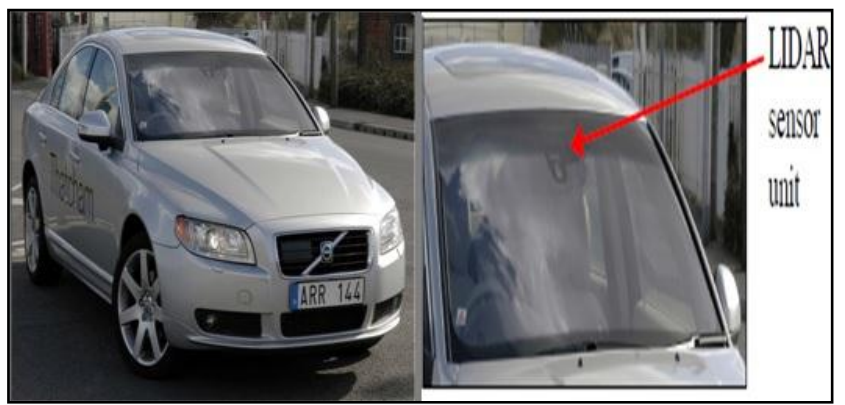

Fig.6: LIDAR Sensor Unit
Participants were loaned the test vehicle shown in Figure 3 for a period of up to one week to allow familiarisation with the controls. The test car was an S80 loaned by Volvo that was retro-fitted with the City Safety system for purposes of the research. The system is only fitted to new cars, and was launched on the XC60 in November 2008. LIDAR sensor unit. The mileage travelled included an equal split between motorways as well as urban and rural roads, all of which were normal UK roads, for a combined distance of over 20,000 kms. Participants were aged between 25 and 55 years old, and all held full driving licences. During the road driving trials all the 11 drivers had the City Safety system operational, since it could not be deactivated on the test vehicle. For all drivers, no positive interventions of the City Safety system were reported, and no false interventions either. $50 \%$ of drivers reported that they felt safer than usual knowing that they were driving the car fitted with City Safety that had the capability of preventing a low speed collision. $30 \%$ felt no different driving the test vehicle compared to their usual driving. $10 \%$ of drivers felt more confident driving the car fitted with City Safety, and the remaining $10 \%$ felt more nervous. ${ }^{2]}$

\subsection{Result}

In order to identify an impending low speed impact the City Safety system uses a LIDAR sensor mounted in the front windscreen. The car brakes are automatically applied when an imminent collision is identified. The automatic braking can prevent impact under $15 \mathrm{~km} / \mathrm{h}$ and can mitigate an impact between 15 and $30 \mathrm{~km} / \mathrm{h}$ The City Safety system prevents common low speed crashes where whiplash typically occurs. It shows potential for reducing the burden on the wider society as well as insurers. The UK estimates presented indicate the system could affect 351,000 crashes annually by preventing or mitigating the crash. The estimates show that City Safety could also save over 150,000 crashes involving whiplash injuries. This equates to an estimated cost saving of nearly $€ 2$ billion. Studies of driver responses in normal road driving showed no interventions of the system, including no false activations. Collision prevention testing involved drivers driving toward an inflatable target car resulting in automatic application of the brakes to prevent an impact. In these collision assessment tests the majority of drivers felt the instinctive urge to brake in response to the collision situation that was created. Drivers also stated that they understood that the system is designed for emergency situations only and they would not rely upon the system in normal driving. This driver study indicates that driver adaptation to the City Safety system seems unlikely. The City Safety system appears to offer significant benefits to all drivers in preventing the most common sort of impacts. The system islow cost and can be readily made available across a new car fleet. Estimates presented in this paper indicate that significant reductions in injuries and repair costs are 
possible. Due to the late activation of the system in the collision process and the harsh and unpleasant emergency braking applied, an activation of City Safety is expected to discourage drivers from adapting to the technology.

The braking system is a complex arrangement and simply changing one component may completely upset the whole set-up. Pedal ratio, plus bore sizes of the master cylinder, caliper pistons and wheel cylinders are all very critical to maintaining the correct system pressure, and safe braking performance. Weight distribution, tyre size and suspension can all have an effect. Therefore, I decided to do some research. Iknew I wasn't going to get the set-up bang on first time, but at least I wanted to get close. I had read a number of forum pages where builders of Low cost type cars complained of the rear locking up under heavy braking and didn't want the same.

\section{Conclusion}

1. If we can reduced the Driving Interference of Braking and Give the Responsibility To Intelligence Sensor which will Take decision and initiate the Response To give Warning alarm First and if Distance of impact is Closing it will Apply brake Automatically and Stop the Vehicle in advanced.

2. Such that Distraction Driving is a Major Contributor to Accident death, thus by implementing this System we can reduced the Close impact Potential Accident.

3. By dragging the front seat at opposite direction to Impact and increase the Distance And Time Of Direct Impact the death can be minimized and safety of vehicle also can improved, and also it can add the new feature to the car which will attract the customer who prefer safety while traveling.

4. The results of the simulations showed that by using an energy absorbing seating system, crash deceleration can be effectively attenuated and occupant injuries significantly reduced in comparison to conventional seating systems. In future, physical crash tests will still be required as the final certification method for approval of a particular crashworthy mechanical system. However during the development process the application of computer simulation methods as presented in this paper show that it is possible to reduce development costs.

\section{References}

Erik Coelingh, LottaJakobsson, Henrik Lind, Magdalena Lindman (2013) Collision Warning With Auto Brake - A Reallife Safety Perspective, Volvo Car CorporationSweden Paper Number 07-0450.

Matthew Avery, AlixWeeke, Thatcham (2013) Autonomous Braking Systems And Their Potential Effect On Whiplash Injury Reduction, , United Kingdom, Paper Number 09 . 0328.

Implementation Of Autonomous Emergency Braking (AEB), The Next Step In Euro Ncap's Safety Assessment, Richard Schram, Aled Williams, Michiel van Ratingen, European New Car Assessment Programme, Belgium, on behalf of the Euro NCAP P-NCAP Working Group, Paper Number: 130269.

The potential of autonomous emergency braking systems to mitigate passenger vehicle crashes, Australasian Road Safety Research, Policing and Education Conference, Wellington, New Zealand, Doecke S.D., Anderson R.W.G., Mackenzie J.R.R., Ponte G, Centre for Automotive Safety Research

Automatic Emergency Braking (AEB) Report,An Update of the June 2012 Research Report Titled, "Forward-Looking Advanced Braking Technologies Research Report", National Highway Traffic Safety Administration, U.S Department of Transportation

Forward Collision Warning Requirements Report, National Highway Traffic Safety Administration, U.S. Department of Transportation

Andreas Georgi, Marc Zimmermann, Thomas Lich, Lisa Blank, Dr. Nils Kickler, Dr. Reiner Marchthaler, Robert Bosch Corporation(2014), New Approach Of Accident Benefit Analysis For Rear End Collision Avoidance AndMitigation Systems, CR/AEV, P.O.Box 3002 40, 70442 Stuttgart, Germany, Paper Number 09-0281.

The Role of Driver Distraction in Traffic Crashes, AAA Foundation for Traffic Safety, May 2001, http://www.aaafoundation.org/pdf/distraction.pdf

Hosking, S., Young, K. \& Regan, M. (2007). The effects of text messaging on young novice driver performance. In: I.J. Faulks, M. Regan, M. Stevenson, J. Brown, A. Porter \& J.D. Irwin (Eds.)

Luciano Alonso, Vicente Milanés, Carlos Torre-Ferrero, Jorge Godoy, Juan P. Oria and Teresa de Pedro, MDPI, Basel, Switzerland. Sensors (2011) Ultrasonic Sensors in Urban Traffic Driving Aid-Systems, 11, 661-673; doi:10.3390/s110100661

Rahul Khade, HarshadMahajan, NitinKedar, Krushna Darwatkar (2013)Automated Braking System, International Journal For Engineering Applications And Technology by. ISSN: 2321-8134 